\title{
LOCAL BEHAVIOR AND GLOBAL EXISTENCE OF POSITIVE SOLUTIONS OF $a u^{\lambda} \leqslant-\Delta u \leqslant u^{\lambda}$
}

\section{COMPORTEMENT LOCAL ET EXISTENCE GLOBALE DES SOLUTIONS POSITIVES DE $a u^{\lambda} \leqslant-\Delta u \leqslant u^{\lambda}$}

\author{
Steven D. TALIAFERRO \\ Mathematics Department, Texas A\&M University, College Station, TX 77843-3368, USA \\ Received 5 December 2000, revised 22 October 2001
}

ABSTRACT. - We study the behavior near the origin of $C^{2}$ positive solutions $u(x)$ of

$$
a u^{\lambda} \leqslant-\Delta u \leqslant u^{\lambda}
$$

in a punctured neighborhood of the origin in $\mathbb{R}^{n}(n>2)$ where the constants $\lambda$ and $a$ satisfy $\frac{n}{n-2}<\lambda<\frac{n+2}{n-2}$ and $0<a<1$. We also study the existence of $C^{2}$ positive solutions of $(*)$ in $\mathbb{R}^{n}$. In both cases we show that changing $a$ from one value in the open interval $(0,1)$ to another value in $(0,1)$ can have a dramatic effect.

(C) 2002 L'Association Publications de l'Institut Henri Poincaré. Published by Elsevier B.V. All rights reserved

RÉSUMÉ. - On étudie le comportement prés de l' origine des solutions positives de classe $C^{2}$ $u(x)$ de

$$
a u^{\lambda} \leqslant-\Delta u \leqslant u^{\lambda}
$$

dans un voisinage épointé de l' origine dans $\mathbb{R}^{n}(n>2)$ où les constantes $\lambda$ et $a$ satisfont $\frac{n}{n-2}<\lambda<\frac{n+2}{n-2}$ et $0<a<1$. On étudie aussi l' existence de solutions positives de classe $C^{2}$ de (*) dans $\mathbb{R}^{n}$. Dans les deux cas nous montrons que changer la valeur de $a$ dans l'intervalle ouvert $(0,1)$ peut avoir un effet dramatique.

(c) 2002 L'Association Publications de l'Institut Henri Poincaré. Published by Elsevier B.V. All rights reserved

\section{Introduction}

Gidas and Spruck [3] prove the following two theorems:

THEOREM A [3]. - If $u(x)$ is a $C^{2}$ positive solution of

$$
-\Delta u=u^{\lambda}
$$

E-mail address: stalia@math.tamu.edu (S.D. Taliaferro). 
in a punctured neighborhood of the origin in $\mathbb{R}^{n}(n>2)$ where the constant $\lambda$ satisfies

$$
\frac{n}{n-2}<\lambda<\frac{n+2}{n-2}
$$

then either $u$ has a removable singularity at the origin or

$$
|x|^{2 /(\lambda-1)} u(x) \rightarrow \ell \quad \text { as }|x| \rightarrow 0^{+}
$$

where $\ell=\ell(n, \lambda)$ is a positive constant.

THEOREM B [3]. - There does not exist a $C^{2}$ positive solution of (1.1) in $\mathbb{R}^{n}(n>2)$ when the constant $\lambda$ satisfies $1 \leqslant \lambda<\frac{n+2}{n-2}$.

In this paper we study the more general problem

$$
a u^{\lambda} \leqslant-\Delta u \leqslant u^{\lambda}
$$

in various subsets of $\mathbb{R}^{n}$ where $a \in(0,1)$. Henceforth we assume $n>2$.

Our two results on the local behavior at the origin of $C^{2}$ positive solutions of (1.3) are

THEOREM 1. - Suppose $\lambda>n /(n-2)$. Then there exists $a=a(n, \lambda) \in(0,1)$ such that for each continuous function $\varphi:(0,1) \rightarrow(0, \infty)$ there exists a $C^{2}$ positive solution $u(x)$ of $(1.3)$ in $\mathbb{R}^{n}-\{0\}$ satisfying

$$
u(x) \neq \mathcal{O}(\varphi(|x|)) \quad \text { as }|x| \rightarrow 0^{+} .
$$

THEOREM 2. - Suppose $\lambda$ satisfies (1.2). Then there exists $a=a(n, \lambda) \in(0,1)$ such that if $u$ is a $C^{2}$ positive solution of (1.3) in a punctured neighborhood of the origin in $\mathbb{R}^{n}$ then

$$
u(x)=\mathcal{O}\left(|x|^{-2 /(\lambda-1)}\right) \quad \text { as }|x| \rightarrow 0^{+}
$$

and $u(x) / \bar{u}(|x|)$ is bounded between positive constants for $|x|$ small and positive where $\bar{u}(r)$ is the average of $u$ on the sphere $|x|=r$.

Our two results on the local behavior at infinity of $C^{2}$ positive solutions of (1.3) are

THEOREM 3. - Suppose $\lambda>n /(n-2)$. Then there exists $a=a(n, \lambda) \in(0,1)$ such that for each continuous function $\varphi:(1, \infty) \rightarrow(0, \infty)$ there exists a $C^{2}$ positive solution $u(x)$ of (1.3) in $\mathbb{R}^{n}$ satisfying

$$
u(x) \neq \mathcal{O}(\varphi(|x|)) \quad \text { as }|x| \rightarrow \infty .
$$

THEOREM 4. - Suppose $\lambda$ satisfies (1.2). Then there exists $a=a(n, \lambda) \in(0,1)$ such that if $u$ is a $C^{2}$ positive solution of (1.3) in the complement of a compact subset of $\mathbb{R}^{n}$ then

$$
u(x)=\mathcal{O}\left(|x|^{-2 /(\lambda-1)}\right) \quad \text { as }|x| \rightarrow \infty
$$

and $u(x) / \bar{u}(|x|)$ is bounded between positive constants for $|x|$ large where $\bar{u}(r)$ is the average of $u$ on the sphere $|x|=r$.

Our two results on the global existence of $C^{2}$ positive solutions of (1.3) are 
THEOREM 5. - Suppose $\lambda>n /(n-2)$. Then there exists $a=a(n, \lambda) \in(0,1)$ such that there exists a $C^{2}$ positive solution $u(x)$ of $(1.3)$ in $\mathbb{R}^{n}$.

THEOREM 6. - Suppose $\lambda$ satisfies (1.2). Then there exists $a=a(n, \lambda) \in(0,1)$ such that there does not exist a $C^{2}$ positive solution $u(x)$ of (1.3) in $\mathbb{R}^{n}$.

When $\lambda$ satisfies (1.2), these six theorems show that changing $a$ from one value in the open interval $(0,1)$ to another value in $(0,1)$ can dramatically affect the local behavior and global existence of $C^{2}$ positive solutions of (1.3).

Note that Theorem 3 implies Theorem 5. However we state Theorem 5 because it nicely complements Theorem 6 and because it facilitates our discussion below.

Theorems 1 and 2 answer two of the seven open questions posed in [8] where we study in a general way $C^{2}$ positive solutions of

$$
a f(u) \leqslant-\Delta u \leqslant f(u)
$$

in a punctured neighborhood of the origin in $\mathbb{R}^{n}$ where $a \in[0,1)$ is a constant and $f:(0, \infty) \rightarrow(0, \infty)$ is a continuous function. Our proof of Theorem 1 in Section 3 is different and shorter than the proofs of two special cases of Theorem 1 given in [8].

Theorem 1 is not true when $\lambda \leqslant n /(n-2)$. In fact we prove in [9] that if $u(x)$ is a $C^{2}$ positive solution of

$$
0 \leqslant-\Delta u \leqslant f(u)
$$

in a punctured neighborhood of the origin in $\mathbb{R}^{n}$ where $f:(0, \infty) \rightarrow(0, \infty)$ is a continuous function satisfying

$$
f(t)=\mathcal{O}\left(t^{n /(n-2)}\right) \quad \text { as } t \rightarrow \infty
$$

then

$$
u(x)=\mathcal{O}\left(|x|^{2-n}\right) \quad \text { as }|x| \rightarrow 0^{+}
$$

and $u(x) / \bar{u}(|x|)$ is bounded between positive constants for $|x|$ small and positive where $\bar{u}(r)$ is the average of $u$ on the sphere $|x|=r$.

When $\lambda=(n+2) /(n-2)$, which is of interest in geometry, we prove in [7] that the conclusion of Theorem 1 is true for each $a \in\left(0,2^{-4 /(n-2)}\right)$ and we conjecture there that the conclusion of Theorem 2 is true for each $a \in\left(2^{-4 /(n-2)}, 1\right)$.

Suppose $\lambda$ satisfies $(1.2)$ and $a \in(0,1)$. Then Gidas and Spruck [3, Theorem 3.3] prove that if $u$ is a $C^{2}$ positive solution of (1.3) in a punctured neighborhood of the origin in $\mathbb{R}^{n}$ such that

$$
h(x):=\frac{-\Delta u(x)}{u(x)^{\lambda}} \quad \text { is } C^{1}
$$

and

$$
|\nabla \log h(x)|=\mathcal{O}\left(\frac{1}{|x|}\right) \quad \text { as }|x| \rightarrow 0^{+}
$$

then either $u$ has a $C^{1}$ extension to the origin or $|x|^{2 /(\lambda-1)} u(x)$ is bounded between positive constants for $|x|$ small and positive. (However we do not understand the third 
sentence of their proof of Theorem 3.3 and we have contacted them for an explanation.) Conditions (1.6) and (1.7) in their result cannot both be omitted when $a$ is near 0 by our Theorem 1. An open question is whether conditions (1.6) and (1.7) can be omitted when $a$ is near 1 . This open question reduces to an ODE problem by our Theorem 2 and the fact that every $C^{2}$ positive bounded solution of (1.3) in a puncture neighborhood of the origin has a $C^{1}$ extension to the origin.

Theorem 3 is not true when $\lambda \leqslant n /(n-2)$. In fact Serrin and Zou [6, Theorem I] prove that if $0 \leqslant \lambda \leqslant n /(n-2)$ then there does not exist a $C^{2}$ positive solution of

$$
-\Delta u \geqslant u^{\lambda} \quad \text { in } \mathbb{R}^{n}-B_{1}(0)
$$

and this result can be easily extended to include all negative values of $\lambda$.

Consider the

Question. - For what values of $\lambda \in \mathbb{R}$ and $a \in(0,1]$ does there exist a $C^{2}$ positive solution of (1.3) in $\mathbb{R}^{n}$ ?

To answer this question we consider three mutually exclusive possibilities for the value of $\lambda$.

Case I. Suppose $-\infty<\lambda \leqslant n /(n-2)$. Then for each $a \in(0,1],(1.3)$ does not have a $C^{2}$ positive solution in $\mathbb{R}^{n}$ by Serrin and Zou's result mentioned above.

Case II. Suppose $\lambda$ satisfies (1.2). Then our Theorems 5 and 6 hold.

Case III. Suppose $(n+2) /(n-2) \leqslant \lambda<\infty$. Then for each $a \in(0,1],(1.3)$ has a $C^{2}$ positive solution in $\mathbb{R}^{n}$ because Fowler [2] shows that (1.3) with $a=1$ has a $C^{2}$ positive radial solution in $\mathbb{R}^{n}$.

For $\lambda$ satisfying (1.2) and for $j=1,2, \ldots, 6$, let $I_{j}=I_{j}(n, \lambda)$ be the set of all $a \in(0,1)$ such that the conclusion of Theorem $j$ is true. By Theorems $1-6$,

(i) $I_{j}$ is a nonempty subinterval of $(0,1)$ for $j=1,2, \ldots, 6$.

(ii) The left endpoint of each of the intervals $I_{1}, I_{3}$, and $I_{5}$, is 0 .

(iii) The right endpoint of each of the intervals $I_{2}, I_{4}$, and $I_{6}$, is 1 .

(iv) $I_{1} \cap I_{2}=I_{3} \cap I_{4}=I_{5} \cap I_{6}=\emptyset$.

(v) $I_{5} \cup I_{6}=(0,1)$ and $I_{3} \subset I_{5}$.

Some interesting open questions are:

(i) Is $I_{1} \cup I_{2}=(0,1)$ (respectively $\left.I_{3} \cup I_{4}=(0,1)\right)$ ? If not, what is the local behavior at the origin (respectively at infinity) of $C^{2}$ positive solutions of (1.3) when $a \in(0,1)-\left(I_{1} \cup I_{2}\right)$ (respectively $\left.a \in(0,1)-\left(I_{3} \cup I_{4}\right)\right)$ ?

(ii) Is $I_{1}=I_{3}=I_{5}$ ?

(iii) Is $I_{2}=I_{4}=I_{6}$ ?

(iv) Which of the intervals $I_{j}, j=1,2, \ldots, 6$, are open?

With regard to question (iii) we have

THEOREM 7. - Suppose $\lambda$ satisfies (1.2). Then

$$
I_{2}^{\lambda} \supset I_{6} \quad \text { and } \quad I_{4}^{\lambda} \supset I_{6}
$$

where we define $I^{\lambda}=\left\{a^{\lambda}: a \in I\right\}$. 


\section{Preliminary lemmas}

For later we need two lemmas.

Lemma 1. - Suppose, for $j=1,2, \ldots$, there exists a $C^{2}$ positive solution $u_{j}(x)$ of

$$
\begin{aligned}
& a_{j}|x|^{-\sigma} u_{j}^{\lambda} \leqslant-\Delta u_{j} \leqslant|x|^{-\sigma} u_{j}^{\lambda} \quad \text { in } \Gamma_{j} \\
& u_{j}(x) \neq \mathcal{O}\left(|x|^{-(2-\sigma) /(\lambda-1)}\right) \quad \text { as }|x| \rightarrow 0^{+}
\end{aligned}
$$

where $\Gamma_{j}$ is a punctured neighborhood of the origin in $\mathbb{R}^{n}(n>2), \lambda>n /(n-2)$ and $\sigma \in[0,2)$ are constants, and the sequence $\left\{a_{j}\right\}_{j=1}^{\infty} \subset(0,1]$ converges to $a \in(0,1]$. Then there exists a $C^{2}$ positive solution $v$ of

$$
a^{\lambda} v^{\lambda} \leqslant-\Delta v \leqslant v^{\lambda} \quad \text { in } \mathbb{R}^{n}
$$

When $\sigma=0$ and $a_{j} \equiv 1$, Lemma 1 is well-known and easy to prove using a blow-up argument of Schoen [5]. See also Chen and Lin [1, p. 982]. However when $a \in(0,1)$, $C^{2}$ regularity is lost at a critical point in their proofs and new methods are needed.

Proof of Lemma 1. - Choose positive constants $r_{j}$ such that $B_{2 r_{j}}(0) \subset \Gamma_{j} \cup\{0\}$ and let $\Omega_{j}=B_{r_{j}}(0)$. Since $u_{j}$ is positive and superharmonic in $\Gamma_{j}$, it is well-known (see for example [4]) that

$$
u_{j},-\Delta u_{j} \in L^{1}\left(\Omega_{j}\right)
$$

and that there exist a nonnegative constant $m_{j}$ and a continuous function $h_{j}: \bar{\Omega}_{j} \rightarrow \mathbb{R}$ which is harmonic in $\Omega_{j}$ such that

$$
u_{j}(x)=\frac{m_{j}}{|x|^{n-2}}+\alpha_{n} \int_{\Omega_{j}} \frac{-\Delta u_{j}(y)}{|x-y|^{n-2}} \mathrm{~d} y+h_{j}(x) \quad \text { for } x \in \bar{\Omega}_{j}-\{0\} .
$$

Here $\alpha_{n}=\frac{1}{n(n-2) \omega_{n}}$ where $\omega_{n}$ is the volume of the unit ball in $\mathbb{R}^{n}$. It follows from (2.3), (2.1a), and the nonnegativity of $\sigma$ that $u_{j}^{\lambda} \in L^{1}\left(\Omega_{j}\right)$. Thus, since $\lambda>n /(n-2)$ we have $m_{j}=0$.

Let $\left\{\varepsilon_{j}\right\}_{j=1}^{\infty} \subset(0,1)$ be a sequence which converges to 0 . Choose $\bar{x}_{j} \in \frac{1}{2} \Omega_{j}-\{0\}$ such that $\lim _{j \rightarrow \infty}\left|\bar{x}_{j}\right|=0$,

$$
\begin{aligned}
& \lim _{j \rightarrow \infty}\left|\varepsilon_{j} \bar{x}_{j}\right|^{2 /(\lambda-1)}\left|\bar{x}_{j}\right|^{-\sigma /(\lambda-1)} u_{j}\left(\bar{x}_{j}\right)=\infty, \quad \text { and } \\
& \max _{\bar{\Omega}_{j}}\left|h_{j}\right|<\left|\bar{x}_{j}\right|^{-(2-\sigma) /(\lambda-1)} .
\end{aligned}
$$

Following Schoen [5], let $B_{j}=B_{\varepsilon_{j}\left|\bar{x}_{j}\right|}\left(\bar{x}_{j}\right)$ and define $\varphi_{j}: \bar{B}_{j} \rightarrow[0, \infty)$ by

$$
\varphi_{j}(x)=u_{j}(x) d_{j}(x)^{2 /(\lambda-1)}|x|^{-\sigma /(\lambda-1)} \quad \text { where } d_{j}(x)=\operatorname{dist}\left\{x, \partial B_{j}\right\} .
$$

Choose $x_{j} \in B_{j}$ such that $\varphi_{j}\left(x_{j}\right)=\max _{\bar{B}_{j}} \varphi_{j}$. Then

$$
\varphi_{j}\left(x_{j}\right) \geqslant \varphi_{j}\left(\bar{x}_{j}\right) \rightarrow \infty \quad \text { as } j \rightarrow \infty
$$


by (2.5).

Making the change of variables

$$
w_{j}(\xi)=\frac{u_{j}(x)}{u_{j}\left(x_{j}\right)}, \quad x=\left|x_{j}\right|^{\sigma / 2} u_{j}\left(x_{j}\right)^{-(\lambda-1) / 2} \xi+x_{j}
$$

in (2.4) we obtain

$$
w_{j}(\xi)=\alpha_{n} \int_{\Sigma_{j}} \frac{-\Delta w_{j}(\zeta)}{|\xi-\zeta|^{n-2}} \mathrm{~d} \zeta+H_{j}(\xi) \quad \text { for } \xi \in \bar{\Sigma}_{j}-\left\{\xi_{j}\right\}
$$

where $\Sigma_{j}$ is the set of all $\xi \in \mathbb{R}^{n}$ such that $x$, as given by (2.7), is in $\Omega_{j}$ and where $\xi_{j}$ is the inverse image of $x=0$ in (2.7) and where

$$
H_{j}(\xi)=\frac{h_{j}(x)}{u_{j}\left(x_{j}\right)} .
$$

By (2.5) and (2.6) we have

$$
\max _{\bar{\Sigma}_{j}}\left|H_{j}\right|<\frac{\left(1-\varepsilon_{j}\right)^{-\sigma /(\lambda-1)}}{\varphi_{j}\left(x_{j}\right)} \rightarrow 0 \quad \text { as } j \rightarrow \infty .
$$

Note that the ball given by $\left|x-x_{j}\right|<\frac{1}{2} d_{j}\left(x_{j}\right)$ maps under the transformation (2.7) onto the ball $|\xi|<\rho_{j}$ where

$$
\rho_{j}:=\frac{1}{2} \varphi_{j}\left(x_{j}\right)^{(\lambda-1) / 2} \rightarrow \infty \quad \text { as } j \rightarrow \infty
$$

and that

$$
\begin{aligned}
w_{j}(\xi) & =\frac{\varphi_{j}(x)}{\varphi_{j}\left(x_{j}\right)}\left(\frac{d_{j}\left(x_{j}\right)}{d_{j}(x)}\right)^{2 /(\lambda-1)}\left(\frac{|x|}{\left|x_{j}\right|}\right)^{\sigma /(\lambda-1)} \\
& <2^{2 /(\lambda-1)}\left(\frac{1+\varepsilon_{j}}{1-\varepsilon_{j}}\right)^{\sigma /(\lambda-1)} \text { for }|\xi|<\rho_{j}
\end{aligned}
$$

because $d_{j}(x)>\frac{1}{2} d_{j}\left(x_{j}\right)$ for $\left|x-x_{j}\right|<\frac{1}{2} d_{j}\left(x_{j}\right)$. Also by (2.1a) we have

$$
\left(\frac{1-\varepsilon_{j}}{1+\varepsilon_{j}}\right)^{\sigma} a_{j} w_{j}^{\lambda} \leqslant-\Delta w_{j} \leqslant\left(\frac{1+\varepsilon_{j}}{1-\varepsilon_{j}}\right)^{\sigma} w_{j}^{\lambda} \text { for }|\xi|<\rho_{j} .
$$

It follows therefore from standard elliptic theory that there exists a continuously differentiable function $w: \mathbb{R}^{n} \rightarrow[0, \infty)$ such that a subsequence of $w_{j}$ (which we again denote by $w_{j}$ ) converges uniformly on compact subsets of $\mathbb{R}^{n}$ to $w$. (However unless $a=1$ we cannot conclude that $w$ is $C^{2}$.) 
Letting $j \rightarrow \infty$ in (2.8) and using (2.9), (2.10), and Fatou's lemma we get

$$
w(\xi) \geqslant \alpha_{n} \int_{\mathbb{R}^{n}} \frac{a w(\zeta)^{\lambda}}{|\zeta-\xi|^{n-2}} \mathrm{~d} \zeta \quad \text { for } \xi \in \mathbb{R}^{n} .
$$

Thus $\liminf _{|\zeta| \rightarrow \infty} w(\zeta)=0$ for otherwise the integral in (2.11) would be infinite for each $\xi \in \mathbb{R}^{n}$.

We now show that (2.11) holds with the inequality reversed and the factor $a$ omitted. Let $\xi_{1} \in \mathbb{R}^{n}$ and let $\varepsilon>0$. Choose $\xi_{2} \in \mathbb{R}^{n}$ such that $w\left(\xi_{2}\right)<\varepsilon / 2^{n-1}$ and then choose $r>0$ such that

$$
\left|\zeta-\xi_{2}\right| \leqslant 2\left|\zeta-\xi_{1}\right| \text { for }|\zeta|>r .
$$

For $j$ large enough that $B_{r}(0) \subset \Sigma_{j}-\left\{\xi_{j}\right\}$ we have by (2.8) that

$$
w_{j}\left(\xi_{1}\right)=\alpha_{n} \int_{|\zeta|<r} \frac{-\Delta w_{j}(\zeta) \mathrm{d} \zeta}{\left|\zeta-\xi_{1}\right|^{n-2}}+I_{j}+H_{j}\left(\xi_{1}\right)
$$

where

$$
\begin{aligned}
I_{j} & :=\alpha_{n} \int_{\Sigma_{j}-B_{r}(0)} \frac{-\Delta w_{j}(\zeta) \mathrm{d} \zeta}{\left|\zeta-\xi_{1}\right|^{n-2}} \\
& \leqslant 2^{n-2}\left[w_{j}\left(\xi_{2}\right)-H_{j}\left(\xi_{2}\right)\right], \quad \text { by (2.12) and (2.8) } \\
& \leqslant \varepsilon, \quad \text { for } j \text { large }
\end{aligned}
$$

by (2.9). Thus letting $j \rightarrow \infty$ in (2.13) and using (2.10) we find

$$
w\left(\xi_{1}\right) \leqslant \alpha_{n} \int_{|\zeta|<r} \frac{w(\zeta)^{\lambda} \mathrm{d} \zeta}{\left|\zeta-\xi_{1}\right|^{n-2}}+\varepsilon \leqslant \alpha_{n} \int_{\mathbb{R}^{n}} \frac{w(\zeta)^{\lambda} \mathrm{d} \zeta}{\left|\zeta-\xi_{1}\right|^{n-2}}+\varepsilon
$$

Since $\varepsilon>0$ was arbitrary it can be omitted in (2.14). Thus letting

$$
v(\xi)=\alpha_{n} \int_{\mathbb{R}^{n}} \frac{w(\zeta)^{\lambda} \mathrm{d} \zeta}{|\zeta-\xi|^{n-2}}
$$

we have by (2.11) that $v$ is a $C^{2}$ nonnegative solution of (2.2). By (2.7), $w(0)=1$ and thus $v$ is positive on $\mathbb{R}^{n}$.

Lemma 2. - Suppose, for $j=1,2, \ldots$, there exists a $C^{2}$ positive solution $v_{j}(y)$ of

$$
\begin{aligned}
& a_{j} v_{j}^{\lambda} \leqslant-\Delta v_{j} \leqslant v_{j}^{\lambda} \quad \text { in } \Omega_{j} \\
& v_{j}(y) \neq \mathcal{O}\left(|y|^{-2 /(\lambda-1)}\right) \quad \text { as }|y| \rightarrow \infty
\end{aligned}
$$

where $\Omega_{j}$ is the complement of a compact subset of $\mathbb{R}^{n}(n>2)$, $\lambda$ is a constant satisfying (1.2), and the sequence $\left\{a_{j}\right\}_{j=1}^{\infty} \subset(0,1]$ converges to $a \in(0,1]$. Then there exists a $C^{2}$ positive solution $v$ of (2.2). 
Proof. - Let $u_{j}(x)=|y|^{n-2} v_{j}(y)$ where $y=x /|x|^{2}$. (This is the Kelvin transform.) Then $u_{j}(x)$ is a $C^{2}$ positive solution of $(2.1 \mathrm{a}, \mathrm{b})$ where $\sigma=n+2-\lambda(n-2) \in(0,2)$ and

$$
\Gamma_{j}=\left\{x \in \mathbb{R}^{n}: x /|x|^{2} \in \Omega_{j}\right\}
$$

is a punctured neighborhood of the origin. Thus Lemma 2 follows from Lemma 1.

\section{Proofs}

In this section we prove Theorems 1-7.

Proof of Theorem 1. - Define $W, w: \mathbb{R}^{n} \rightarrow \mathbb{R}$ by

$$
W(y)= \begin{cases}1, & \text { for }|y|<1 \\ \frac{1}{|y|^{n-2}}, & \text { for }|y| \geqslant 1\end{cases}
$$

and $w=N\left(W^{\lambda}\right)$ where $N$ is the Newtonian potential operator over $\mathbb{R}^{n}$. Then $w(y)$ equals

$$
\frac{1}{n-2}\left[\left(\frac{1}{2}+\frac{1}{\lambda(n-2)-2}\right)-\left(\frac{1}{2}-\frac{1}{n}\right)|y|^{2}\right]
$$

or

$$
\frac{1}{n-2} \frac{1}{|y|^{n-2}}\left[\left(\frac{1}{n}+\frac{1}{\lambda(n-2)-n}\right)-\left(\frac{1}{\lambda(n-2)-n}-\frac{1}{\lambda(n-2)-2}\right) \frac{1}{|y|^{\lambda(n-2)-n}}\right]
$$

depending on whether $|y|<1$ or $|y| \geqslant 1$ respectively. Hence

$$
C_{1} \leqslant \frac{W(y)}{w(y)} \leqslant C_{2} \quad \text { for } y \in \mathbb{R}^{n}
$$

where $C_{1}=C_{1}(n, \lambda)$ and $C_{2}=C_{2}(n, \lambda)$ are positive constants whose values will not change from line to line.

For each $h>0$ define $V_{h}, v_{h}: \mathbb{R}^{n} \rightarrow \mathbb{R}$ by $V_{h}(x)=K W(x / h)$, where $K=K(h)$ satisfies $K^{\lambda-1} h^{2}=1$, and $v_{h}=N\left(V_{h}^{\lambda}\right)$. Then

$$
v_{h}(x)=K w\left(\frac{x}{h}\right) \quad \text { for } x \in \mathbb{R}^{n} .
$$

Thus by (3.1)

$$
C_{1} \leqslant \frac{V_{h}(x)}{v_{h}(x)} \leqslant C_{2} \quad \text { for } x \in \mathbb{R}^{n} \quad \text { and } \quad h>0 .
$$

For later note that

$$
V_{h}(x)= \begin{cases}K, & \text { for }|x|<h \\ \frac{L}{|x|^{n-2}}, & \text { for }|x| \geqslant h\end{cases}
$$


where $L=L(h)$ satisfies

$$
L^{\lambda-1}=h^{\lambda(n-2)-n} .
$$

Let $\varepsilon$ be a positive constant and let $\varphi:(0,1) \rightarrow(0, \infty)$ be a continuous function. Let $U_{0}=W$ and let $\left\{\varepsilon_{j}\right\}_{j=1}^{\infty} \subset(0,1)$ be a sequence such that

$$
\sum_{j=1}^{\infty} \varepsilon_{j}=\varepsilon
$$

Since, by (3.3) and (3.4),

$$
\lim _{h \rightarrow 0^{+}} \sup _{\left|x-x^{j}\right| \geqslant r_{j}} \frac{V_{h}\left(x-x^{j}\right)}{U_{0}(x)}=0 \quad \text { for } j=1,2, \ldots,
$$

where $x^{j}=\left(2^{-2 j}, 0, \ldots, 0\right) \in \mathbb{R}^{n}$ and $r_{j}=2^{-2 j-1}$, there exists $h_{j} \in\left(0, r_{j}\right), j=1$, $2, \ldots$, such that

$$
\begin{aligned}
& \sum_{j=1}^{\infty} L_{j}^{\lambda}<\infty \quad \text { where } L_{j}=L\left(h_{j}\right)=h_{j}^{(\lambda(n-2)-n) /(\lambda-1)}, \\
& \lim _{j \rightarrow \infty} \frac{K_{j}}{\varphi\left(\left|x^{j}\right|\right)}=\infty \quad \text { where } K_{j}=K\left(h_{j}\right)=h_{j}^{-2 /(\lambda-1)},
\end{aligned}
$$

and

$$
U_{j}(x) \leqslant \frac{C_{1}}{C_{2}} \varepsilon_{j} U_{0}(x) \text { for }\left|x-x^{j}\right| \geqslant r_{j} \text { and } j=1,2, \ldots
$$

where $U_{j}(x)=V_{h_{j}}\left(x-x^{j}\right)$ for $j=1,2, \ldots$.

Letting $u_{j}=N\left(U_{j}^{\lambda}\right), j=0,1, \ldots$, and noting that (3.2) and (3.1) imply

$$
C_{1} \leqslant \frac{U_{j}(x)}{u_{j}(x)} \leqslant C_{2} \quad \text { for } x \in \mathbb{R}^{n} \text { and } j=0,1, \ldots
$$

it follows from (3.8) that

$$
u_{j} \leqslant \frac{1}{C_{1}} U_{j} \leqslant \frac{\varepsilon_{j}}{C_{2}} U_{0} \leqslant \varepsilon_{j} u_{0} \quad \text { on } \mathbb{R}^{n}-B_{r_{j}}\left(x^{j}\right)
$$

for $j=1,2, \ldots$

Let $r_{0}>0$. Choose a positive integer $j_{0}$ such that $\left|x^{j_{0}}\right|+r_{j_{0}}<r_{0}$. Then for $j \geqslant j_{0}$ and $|x|>2 r_{0}$ we see by (3.3) that

$$
U_{j}(x)^{\lambda}=V_{h_{j}}\left(x-x^{j}\right)^{\lambda}=\frac{L_{j}^{\lambda}}{\left|x-x^{j}\right|^{(n-2) \lambda}} \leqslant \frac{L_{j}^{\lambda}}{r_{0}^{(n-2) \lambda}}
$$

and

$$
\left|\nabla\left(U_{j}(x)^{\lambda}\right)\right|=\frac{(n-2) \lambda L_{j}^{\lambda}}{\left|x-x^{j}\right|^{1+(n-2) \lambda}} \leqslant \frac{(n-2) \lambda L_{j}^{\lambda}}{r_{0}^{(n-2) \lambda+1}} .
$$


Thus by (3.6), $\sum_{j=j_{0}}^{\infty} U_{j}(x)^{\lambda}$ is $C^{1}$ and uniformly Lipschitz continuous on $|x|>2 r_{0}$. Hence $\sum_{j=0}^{\infty} U_{j}(x)^{\lambda}$ is uniformly Lipschitz continuous on $|x|>\rho$ for each $\rho>0$.

Also by the monotone convergence theorem,

$$
u:=N\left(\sum_{j=0}^{\infty} U_{j}^{\lambda}\right)=\sum_{j=0}^{\infty} N\left(U_{j}^{\lambda}\right)=\sum_{j=0}^{\infty} u_{j} \quad \text { in } \mathbb{R}^{n}-\{0\}
$$

which by (3.10) and (3.5) implies that $u$ is finite at each point of $\mathbb{R}^{n}-\{0\}$. Hence $u$ is $C^{2}$ on $\mathbb{R}^{n}-\{0\}$ and

$$
-\Delta u=\sum_{j=0}^{\infty} U_{j}^{\lambda} \leqslant \sum_{j=0}^{\infty} C_{2}^{\lambda} u_{j}^{\lambda} \leqslant C_{2}^{\lambda} u^{\lambda} \quad \text { in } \mathbb{R}^{n}-\{0\} .
$$

Furthermore, it follows from (3.12), (3.9), and (3.7) that

$$
\frac{u\left(x^{j}\right)}{\varphi\left(\left|x^{j}\right|\right)} \geqslant \frac{u_{j}\left(x^{j}\right)}{\varphi\left(\left|x^{j}\right|\right)} \geqslant \frac{1}{C_{2}} \frac{U_{j}\left(x^{j}\right)}{\left.\varphi\left|x^{j}\right|\right)}=\frac{1}{C_{2}} \frac{K_{j}}{\varphi\left(\left|x^{j}\right|\right)} \rightarrow \infty \quad \text { as } j \rightarrow \infty .
$$

To complete the proof of Theorem 1, we now show

$$
-\Delta u \geqslant \frac{C_{1}^{\lambda}}{(1+\varepsilon)^{\lambda} 2^{\lambda-1}} u^{\lambda} \quad \text { in } \mathbb{R}^{n}-\{0\} .
$$

First by (3.12), (3.10), (3.5), (3.9), and (3.13) we have in $\mathbb{R}^{n}-\{0\}-\bigcup_{j=1}^{\infty} B_{r_{j}}\left(x^{j}\right)$ that

$$
C_{1}^{\lambda} u^{\lambda} \leqslant C_{1}^{\lambda}\left(u_{0}+\varepsilon u_{0}\right)^{\lambda} \leqslant(1+\varepsilon)^{\lambda} U_{0}^{\lambda} \leqslant(1+\varepsilon)^{\lambda}(-\Delta u) .
$$

Next let $j$ be a fixed positive integer. Then by (3.10),

$$
u_{i} \leqslant \varepsilon_{i} u_{0} \quad \text { in } B_{r_{j}}\left(x^{j}\right) \text { for } i \notin\{0, j\} .
$$

Thus by (3.12), (3.5), (3.9), and (3.13) we have in $B_{r_{j}}\left(x^{j}\right)$ that

$$
\begin{aligned}
C_{1}^{\lambda} u^{\lambda} & \leqslant C_{1}^{\lambda}\left(u_{0}+u_{j}+\varepsilon u_{0}\right)^{\lambda} \leqslant C_{1}^{\lambda}(1+\varepsilon)^{\lambda}\left(u_{0}+u_{j}\right)^{\lambda} \\
& \leqslant C_{1}^{\lambda}(1+\varepsilon)^{\lambda} 2^{\lambda-1}\left(u_{0}^{\lambda}+u_{j}^{\lambda}\right) \leqslant(1+\varepsilon)^{\lambda} 2^{\lambda-1}\left(U_{0}^{\lambda}+U_{j}^{\lambda}\right) \\
& \leqslant(1+\varepsilon)^{\lambda} 2^{\lambda-1}(-\Delta u) .
\end{aligned}
$$

Hence (3.14) holds.

Remark. - The proof of Theorem 1 gives an explicit expression for $a(n, \lambda)$. Indeed, letting

$$
\begin{aligned}
& A_{1}=\frac{1}{2}+\frac{1}{\lambda(n-2)-2}, \quad A_{2}=\frac{1}{n}+\frac{1}{\lambda(n-2)-2}, \quad \text { and } \\
& A_{3}=\frac{1}{n}+\frac{1}{\lambda(n-2)-n},
\end{aligned}
$$


the optimal choices for $C_{1}(n, \lambda)$ and $C_{2}(n, \lambda)$ in (3.1) are

$$
C_{1}=\frac{n-2}{\max \left\{A_{1}, A_{3}\right\}} \quad \text { and } \quad C_{2}=\frac{n-2}{A_{2}}
$$

and the proof of Theorem 1 shows that $a=a(n, \lambda)$ in the statement of Theorem 1 can be taken to be any positive number less than

$$
\frac{C_{1}^{\lambda}}{C_{2}^{\lambda} 2^{\lambda-1}}=\frac{A_{2}^{\lambda}}{2^{\lambda-1} \max \left\{A_{1}^{\lambda}, A_{3}^{\lambda}\right\}} .
$$

Proof of Theorem 2. - Suppose for contradiction that for each constant $a \in(0,1)$ there exists a $C^{2}$ positive solution $u(x)$ of (1.3) in a punctured neighborhood of the origin in $\mathbb{R}^{n}$ such that (1.4) does not hold. Then letting $\left\{a_{j}\right\}_{j=1}^{\infty} \subset(0,1)$ be any sequence which converges to 1 , we have by Lemma 1 with $\sigma=0$ that there exists a $C^{2}$ positive solution of (1.1) in $\mathbb{R}^{n}$ which contradicts Theorem B in the introduction.

Let $u(x)$ be a $C^{2}$ positive solution of (1.3) in a punctured neighborhood of the origin in $\mathbb{R}^{n}$ which satisfies (1.4). To complete the proof of Theorem 2 , it remains only to show that $u(x) / \bar{u}(|x|)$ is bounded between positive constants for $|x|$ small and positive, and this is indeed the case by the asymptotic Harnack inequality (see Veron [10, Lemma 1.5]) and the fact that

$$
0<\frac{-\Delta u(x)}{u(x)} \leqslant u(x)^{\lambda-1}=\mathcal{O}\left(|x|^{-2}\right) \quad \text { as }|x| \rightarrow 0^{+} .
$$

Proof of Theorem 3. - A proof of Theorem 3 can be obtained by making the following changes to the proof of Theorem 1. The first change occurs above (3.6) where we now define $x^{j}=\left(2^{2 j}, 0, \ldots, 0\right)$ and $r_{j}=1$. The next change is to replace the paragraph containing equation (3.11) with the following paragraph.

Let $r_{0}>1$. Choose a positive integer $j_{0}$ such that $\left|x^{j_{0}}\right|-1>r_{0}$. Then for $j \geqslant j_{0}$ and $|x|<r_{0}$ we see by (3.3) that

$$
U_{j}(x)^{\lambda}=V_{h_{j}}\left(x-x^{j}\right)^{\lambda}=\frac{L_{j}^{\lambda}}{\left|x-x^{j}\right|^{(n-2) \lambda}} \leqslant L_{j}^{\lambda}
$$

and

$$
\left|\nabla\left(U_{j}(x)^{\lambda}\right)\right|=\frac{(n-2) \lambda L_{j}^{\lambda}}{\left|x-x^{j}\right|^{1+(n-2) \lambda}} \leqslant(n-2) \lambda L_{j}^{\lambda} .
$$

Thus by (3.6), $\sum_{j=j_{0}}^{\infty} U_{j}(x)^{\lambda}$ is $C^{1}$ and uniformly Lipschitz continuous on $|x|<r_{0}$. Hence $\sum_{j=0}^{\infty} U_{j}(x)^{\lambda}$ is uniformly Lipschitz continuous on $|x|<\rho$ for each $\rho>0$.

Finally, in the remainder of the proof of Theorem 1 replace each occurrence of $\mathbb{R}^{n}-\{0\}$ with $\mathbb{R}^{n}$.

Proof of Theorem 4. - The proof of Theorem 4 is basically the same as the proof of Theorem 2 except we use Lemma 2 instead of Lemma 1. We omit the details. 
Proof of Theorem 5. - Theorem 5 has three short proofs. First, Theorem 3 implies Theorem 5. Second, by Theorem 1 there exists a constant $a \in(0,1)$ such that $(1.3)$ has a $C^{2}$ positive solution $u$ in $\mathbb{R}^{n}-\{0\}$ which does not satisfy (1.4). Applying Lemma 1 with $\sigma=0$ and with $a_{j}=a$ and $u_{j}=u$ for $j=1,2, \ldots$, we obtain Theorem 5. And third, a $C^{2}$ positive solution of (1.3) in $\mathbb{R}^{n}$ with $a=\left(C_{1} / C_{2}\right)^{\lambda}$ is $u=C_{2}^{\lambda /(\lambda-1)} w$, where $C_{1}, C_{2}$, and $w$ are as defined in the first paragraph of the proof of Theorem 1.

Proof of Theorem 6. - Suppose for contradiction there exists a sequence $\left\{a_{j}\right\}_{j=1}^{\infty} \subset$ $(0,1)$ such that $\lim _{j \rightarrow \infty} a_{j}=1$ and a sequence $\left\{u_{j}: \mathbb{R}^{n} \rightarrow(0, \infty)\right\}_{j=1}^{\infty}$ of $C^{2}$ functions satisfying

$$
a_{j} u_{j}^{\lambda} \leqslant-\Delta u_{j} \leqslant u_{j}^{\lambda} \quad \text { in } \mathbb{R}^{n} .
$$

It follows then from Lemma 2 and Theorem B that (after omitting a finite number of terms of the sequence $u_{j}$ )

$$
u_{j}(x)=\mathcal{O}\left(|x|^{-2 /(\lambda-1)}\right) \quad \text { as }|x| \rightarrow \infty .
$$

Thus $u_{j}$ assumes its maximum at some $x_{j} \in \mathbb{R}^{n}$. By translating and scaling each $u_{j}$ we can assume $x_{j}=0$ and

$$
u_{j}(0)=1=\max _{\mathbb{R}^{n}} u_{j} .
$$

It follows from (3.15), (3.17), and standard elliptic theory that there exists a continuously differentiable function $u: \mathbb{R}^{n} \rightarrow[0, \infty)$ such that a subsequence of $u_{j}$ (which we again denote by $u_{j}$ ) converges uniformly on compact subsets of $\mathbb{R}^{n}$ to $u$. Thus multiplying (3.15) by a nonnegative test function $\varphi \in C_{0}^{\infty}\left(\mathbb{R}^{n}\right)$, integrating over $\mathbb{R}^{n}$, integrating by parts, and letting $j \rightarrow \infty$ we obtain

$$
u^{\lambda} \leqslant-\Delta u \leqslant u^{\lambda} \quad \text { in } \mathcal{D}^{\prime}\left(\mathbb{R}^{n}\right) .
$$

Hence $-\Delta u=u^{\lambda}$ in $\mathcal{D}^{\prime}\left(\mathbb{R}^{n}\right)$ and this together with the fact that $u$ is $C^{1}$ implies $u$ is $C^{2}$, which contradicts Theorem B.

Proof of Theorem 7. - Let $\left\{a_{j}\right\}_{j=1}^{\infty} \subset(0,1)-I_{2}$ be a sequence which converges to the left endpoint $A_{2}$ of $I_{2}$. Then by the last paragraph of the proof of Theorem 2 there exists a $C^{2}$ positive solution $u_{j}$ of

$$
a_{j} u_{j}^{\lambda} \leqslant-\Delta u_{j} \leqslant u_{j}^{\lambda}
$$

in a punctured neighborhood $\Gamma_{j}$ of the origin such that

$$
u_{j}(x) \neq \mathcal{O}\left(|x|^{-2 /(\lambda-1)}\right) \quad \text { as }|x| \rightarrow 0^{+} .
$$

Thus by Lemma 1 with $\sigma=0$ we have $A_{2}^{\lambda} \in I_{5}$. Hence

$$
I_{6} \subset\left(A_{2}^{\lambda}, 1\right)=\left(A_{2}, 1\right)^{\lambda} \subset I_{2}^{\lambda} .
$$

Similarly, using Lemma 2 instead of Lemma 1 , we obtain $I_{6} \subset I_{4}^{\lambda}$. 


\section{REFERENCES}

[1] C.-C. Chen, C.-S. Lin, Estimates of the conformal scalar curvature equation via the method of moving planes, Comm. Pure Appl. Math. 50 (1997) 971-1017.

[2] R.H. Fowler, Further studies of Emden's and similar differential equations, Quart. J. Math. Oxford Ser. 2 (1931) 259-288.

[3] B. Gidas, J. Spruck, Global and local behavior of positive solutions of nonlinear elliptic equations, Comm. Pure Appl. Math. 34 (1981) 525-598.

[4] C. Li, Local asymptotic symmetry of singular solutions to nonlinear elliptic equations, Invent. Math. 123 (1996) 221-231.

[5] R. Schoen, On the number of constant scalar curvature metrics in a conformal class, in: H.B. Lawson, K. Tenenblat (Eds.), Differential Geometry: A Symposium in Honor of Manfredo Do Carmo, Wiley, New York, 1991, pp. 311-320.

[6] J. Serrin, H. Zou, Cauchy-Liouville and universal boundedness theorems for quasilinear elliptic equations and inequalities, Preprint.

[7] S.D. Taliaferro, On the growth of superharmonic functions near an isolated singularity I, J. Differential Equations 158 (1999) 28-47.

[8] S.D. Taliaferro, On the growth of superharmonic functions near an isolated singularity II, Comm. Partial Differential Equations 26 (2001) 1003-1026.

[9] S.D. Taliaferro, Isolated singularities of nonlinear elliptic inequalities, Indiana Univ. Math. J. 50 (2001) 1885-1897.

[10] L. Veron, Singular solutions of some nonlinear elliptic equations, Nonlinear Anal. 5 (1981) 225-242. 